\title{
User-centric ambient information systems and applications
}

\author{
Quan Z. Sheng • Elhadi M. Shakshuki • \\ Jiangang Ma
}

Published online: 26 June 2013

(C) Springer-Verlag London 2013

This theme issue looks at new developments in pervasive and ambient systems such as localization, interaction interfaces, context-aware services, smart environments, and personal task scheduling. It is based on selected and rereviewed papers from the 3rd International Conference on Ambient Systems, Networks and Technologies (ANT 2012), which was held at Niagara Falls, Ontario, Canada, August 27-29, 2012. The conference attracted a large number of scientific papers that contributed to the state-ofthe-art in the areas of pervasive and ambient information systems. All the papers selected for this special issue have undergone two rounds of rigorous review process. Based on the reviewers' feedback, seven papers were selected for publication from ten best papers invited from ANT 2012.

The first paper by Adnan, Datta, and MacLean, "Efficient and Accurate Sensor Network Localization", focuses on localization, a key research challenge in wireless sensor networks, and proposes a generic, distributed algorithm that outperforms a typical range-free algorithm Weighted Monte Carlo Localization (WMCL). The paper by Zhou et al. "Innovative Wearable Interfaces: An Exploratory Analysis of Paper-based Interfaces with Camera-Glasses Device Unit" considers ubiquitous interaction approaches and conducts some interesting research by developing a

\footnotetext{
Q. Z. Sheng · J. Ma

School of Computer Science, The University of Adelaide, Adelaide, SA 5005, Australia

e-mail: qsheng@cs.adelaide.edu.au

J. Ma

e-mail: jiangang.ma@adelaide.edu.au

E. M. Shakshuki ( $\square)$

Jodrey School of Computer Science, Acadia University,

Wolfville, NS B4P 2R6, Canada

e-mail: elhadi.shakshuki@acadiau.ca
}

MobilePaperAccess system. This system enables mobile interactions by exploiting a wearable camera-glasses device with a paper-based interface.

Context awareness is a critical feature in user-centric ambient environments in the sense that more personalized services can be offered to users. The following three papers focus on context-awareness and offer some recent developments and advancement. The paper by Ouksel and Lundquist, "A Context-Aware Cross-Layer (CACL) Broadcast Model for Ad Hoc Networks", concerns poor performance of 802.11 medium access control (MAC) and proposes a CACL broadcast model, which offers superior single-hop transmission rates than MAC. Some further extension to CACL is also discussed in the paper. The paper by Yuan and Herbert, "Context-Aware Hybrid Reasoning Framework for Pervasive Healthcare", presents a personalized, flexible, and extensible hybrid reasoning framework for developing context-aware healthcare applications to assist activity of daily living of the elderly. The framework particularly supports context-aware sensor data fusion and anomaly detection by exploiting the hierarchical fuzzy rule-based reasoning (FRBR). The paper by Gronli, Ghinea, and Younas, "Context-Aware and Automatic Configuration of Mobile Devices in Cloud-Enabled Ubiquitous Computing", reports a prototype system on crosssource integration of cloud-based, context aware information in ubiquitous computing.

The paper by Mathew et al., "Building Sustainable Parking Lots with the Web of Things (WoT)", describes a scalable parking-lot infrastructure based on the emerging WoT. With this infrastructure, it is possible to perform mashups of physical things for creating a smart parking spot (SPS) that can be interacted by drivers from the Web. The initial evaluation reveals some promising results such as $40 \%$ of saving in both time and air pollution. The final 
paper by Shakshuki and Hossain, "A Personal Meeting Scheduling Agent", targets meeting scheduling that is a very practical problem in our daily lives. This paper proposes an agent for automated meeting scheduling, with the support of the proposed Personal Meeting Scheduling Protocol (PMSP). Following a structured negotiation protocol influenced by Simultaneous Response Protocol (SRP), PMSP can automatically make decisions and select appropriate negotiation strategies so that possible meeting conflicts can be avoided or resolved.
The guest editors would like to take this opportunity to thank all the authors for the efforts they put in the preparation of their manuscripts and for their valuable contributions. We wish to express our deepest gratitude to the referees who provided very useful and thoughtful feedback to the authors. Finally, our sincere thanks go to the Editorin-Chief, Professor Peter Thomas, of Personal and Ubiquitous Computing for his kind support, advice, and encouragements throughout the preparation of this special issue. 